\title{
Fuzzy homogeneity measures for path-based colour image segmentation
}

\section{J. Chamorro-Martínez, D. Sánchez, B. Prados-Suárez* and E. Galán-Perales}

Department of Computer Science and Artificial Intelligence, University of Granada, C/Periodista Daniel Saucedo Aranda s/n, 18071 Granada, Spain

E-mail: jesus@decsai.ugr.es_E-mail:daniel@decsai.ugr.es

E-mail: belenps@decsai.ugr.es_E-mail: elena@decsai.ugr.es

${ }^{*}$ Corresponding author

\begin{abstract}
In this paper we propose a set of measures to model the concept of homogeneity in path-based image segmentation. We introduce the idea of path homogeneity as the aggregation of resemblances between consecutive pixels in the path. This resemblance is obtained from a measure of resemblance between neighbour pixels. In order to aggregate these resemblance values we propose the use of certain families of $t$-norms that verify a set of intuitive properties. We have studied the performance and behaviour of these functions through a set of experiments. Finally, we have applied these proposals to obtain fuzzy segmentations from real images.
\end{abstract}

Keywords: image segmentation; fuzzy segmentation; path-based segmentation; fuzzy connectivity; fuzzy colour homogeneity.

Reference to this paper should be made as follows: Chamorro-Martínez, J., Sánchez, D., Prados-Suárez, B. and Galán-Perales, E. (2006) 'Fuzzy homogeneity measures for path-based colour image segmentation', Int. J. Intelligent Systems Technologies and Applications, Vol. 1, Nos. 3/4, pp.301-318.

Biographical notes: J. Chamorro-Martínez received the MS and PhD Degrees, both in Computer Science, from the University of Granada in 1995 and 2001 respectively. Since 1996, he is member of the Computer Science Department at the University of Granada where he is now an Associate Professor. His research interests include soft computing image processing, fuzzy logic applied to image characterisation, and information retrieval from image databases.

Daniel Sánchez received his MS Degree in Computer Science in 1995 and his $\mathrm{PhD}$ in Computer Science in 1999, both from the University of Granada. He is an Associate Professor in the Department of Computer Science and Artificial Intelligence of the University of Granada since 2001. He has participated and is currently member of the teams of several projects, and he has published more than 30 papers in international journals and conferences. His current main research interests are in the fields of knowledge discovery and data mining, relational databases, information retrieval, fuzzy sets theory and soft computing.

Belén Prados-Suárez received her MS Degree in Computer Science from the University of Granada, Spain in 2001. She is an Assistant Professor in the 
Department of Computer Science of the University of Jaén since 2004. She has participated and is currently member of the teams of several projects. Her current main research interests are in the fields of fuzzy image processing, analysis and characterisation.

Elena Galán-Perales received her MS Degree in Computer Science from the University of Granada in 2003. Her research interests include soft computing image processing and biomedical image analysis. Currently she is carrying out her $\mathrm{PhD}$ studies and she's participating in the Spanish FIS project Imagen Médica Molecular y Multimodalidad (IM3).

\section{Introduction}

Nowadays, expert systems and intelligent applications, like image database retrieval or robot vision (Chamorro-Martinez et al., 2003a; Ohno and Tsubouchi, 2001), takes as starting point (and even make decisions based on) the results of image segmentation processes, so they should provide the most accurate representation of the content in the image. Therefore, they should represent and model the imprecision and uncertainty in the image so that, following the 'Last commitment principle', all the information will be available in later stages of processing.

Image segmentation consist on performing a partition of the image into connected subsets of pixels, called regions, on the basis of some homogeneity criterion. Many types of segmentation techniques have been proposed in the literature (Cheng et al., 2001; Chen and Lu, 2002), for example those based on histogram analysis (Gillet et al., 2001), clustering (Zhong and Yan, 2000), split and merge (Borges and Aldon, 2000), region growing (Moghaddamzadeh and Bourbakis, 1997), edge-based algorithms (Shiji and Hamada, 1999), and combinations of these techniques (Makrogiannis et al., 2001). Most of the proposals falling in the aforementioned categories, provide a crisp segmentation of images, where each pixel has to belong to a unique region. However, separation between regions is usually imprecise in natural images, as occurs in shadows, bright gradients and colour gradients, and information about this imprecision, needed by intelligent applications, is not often properly modelled by crisp techniques. To solve this problem, some approaches propose the definition of region as a fuzzy subset of pixels, in such a way that every pixel of the image has a membership degree to that region (Cavalho et al., 1999; Chamorro-Martinez et al., 2003b).

The majority of the fuzzy techniques are based on fuzzy clustering, like C-means algorithms, which defines a set of centroids and compute the membership value for all the pixels in the image to each centroid (Yang et al., 2002). Other examples are those based on the definition of fuzzy histograms (Han and Ma, 2002) or fuzzy rule-based systems (Hacbouf and Mezhoud, 2003). Nevertheless, a drawback of most of these fuzzy approaches is that they donot take into account that a region must be topologically connected. As a consequence, pixels belonging to separate and different regions could be assigned to the same cluster.

To face up to the above-mentioned problem, path-based techniques arise which incorporate spatial information related to adjacency between pixels. These approaches are based on the idea of fuzzy topology, introduced by Rosenfeld (1979), and the use of fuzzy connectivity to measure the relationship between any pair of pixels. Thus, given a 
region representative point (called seed point), the path-based methods obtain the associated fuzzy region on the basis of the connectivity between the seed point and the rest of pixels in the image (Moghaddamzadeh and Bourbakis, 1997; Cavalho et al., 1999; Udupa and Samarasekera, 1996; Udupa and Samarasekera, 1996; Philipp-Foliguet et al., 2001; Maeda et al., 2000; Chien and Cheng, 2002).

In the context of path-based image segmentation, an open problem is how to measure the homogeneity (Cheng and Li, 2003) of a 'path' connecting two pixels and, based on this measure, the membership degree of its fuzzy connectivity. Most of the approaches in the literature calculate heterogeneity from the set of distances between consecutive points in the path, the most common solution being the use of a simple aggregation function (sum or maximum) over this set of distances (Moghaddamzadeh and Bourbakis, 1997; Cavalho et al., 1999), and finally obtaining homogeneity as a kind of inverse of heterogeneity. However, to our knowledge, there is not a study about the goodness of different homogeneity functions applied to image segmentation.

In this paper an experimental study of different aggregation functions to calculate homogeneity of a path is performed. To this purpose, a set of desirable properties are defined. In addition, a set of candidate functions that verify these properties are proposed. To analyse the performance of the studied functions, a set of experiments is presented with synthetic and real images. Using the homogeneity functions analysed in the experiment, the region-growing algorithm proposed in Chamorro-Martinez et al. (2003a) is used to obtain fuzzy regions by means of a fuzzy subset of connected pixels.

The rest of the paper is organised as follows: Firstly, in Section 2, we introduce the path-based segmentation techniques. Next, in Section 3, we describe the desirable properties for the aggregation functions and propose some candidates that are also studied in this paper. Finally, the experimental results and the main conclusions are showed in Sections 4 and 5 respectively.

\section{Path-based image segmentation}

Path-based techniques incorporate spatial information related to adjacency between pixels (unlike the clustering-based methods, which classified each pixel without considering its neighbourhood). In these approaches, the segmentation is performed by measuring the connectivity between any pair of pixels as the homogeneity degree of the most homogeneous path joining them (Udupa and Samarasekera, 1996). In a fuzzy approach, given a set of seed points, fuzzy regions are obtained on the basis of the connectivity between each seed point and the rest of pixels in the image (Cavalho et al., 1999; Udupa and Samarasekera, 1996).

In this section we present a path-based methodology to obtain image regions as fuzzy subsets of connected pixels. First, we need to fix the features that characterise a pixel. A fuzzy resemblance relation between neighbour pixels is obtained from a fuzzy resemblance relation between their corresponding feature vectors. This concept is extended later to any pair of pixels following the aforementioned path-based approach. The whole process is particularised for the case of colour features. 


\subsection{Pixel characterisation}

We characterise each pixel $p$ by means of a vector of features $\overrightarrow{f_{p}}$

$$
\overrightarrow{f_{p}}=\left[f_{p}^{1}, f_{p}^{2}, \ldots, f_{p}^{n}\right]
$$

where a feature $f_{p}^{i} \in \mathbb{R}$, with $i \in\{1,2, \ldots, n\}$, is a numerical measure of any relevant characteristic that may be obtained for $p$. As an example of feature vector, let's think about a three-band colour representation like RGB.

In this work we are concerned with segmentation on the basis of colour, i.e., we want to obtain a set of homogeneously coloured regions. Hence, we are interested in colour features.

\subsection{Characterisation by colour}

Although the RGB is the most used model to acquire digital images, it is well known that it is not adequate for colour image segmentation. Instead, other colour spaces based on human perception (HSI, HSV or HLS) seem to be a better choice for this purpose (Russ, 1999). In these spaces, hue $(H)$ represents the colour tone (for example, red or blue), saturation $(S)$ is the amount of colour (for example, bright red or pale red) and the third component (called intensity, value or lightness) is the amount of light (it allows the distinction between a dark colour and a light colour).

In this paper, the HSI colour space will be used (it offers many advantages in a segmentation process, for example, the use of hue avoids the shading effects). Geometrically, this colour space is represented as a cone, in which the axis of the cone is the grey scale progression from black to white, distance from the central axis is the saturation, and the direction is the hue (Figure 1). To calculate the HSI values from the RGB coordinates, the following transform is applied (Russ, 1999):

$$
\begin{aligned}
& H=\arctan \left(\frac{\sqrt{3}(G+B)}{2 R-G-B}\right) \\
& S=1-\min \{R, G, B\} / I \\
& I=(R+G+B) / 3 .
\end{aligned}
$$

Figure 1 HSI colour space

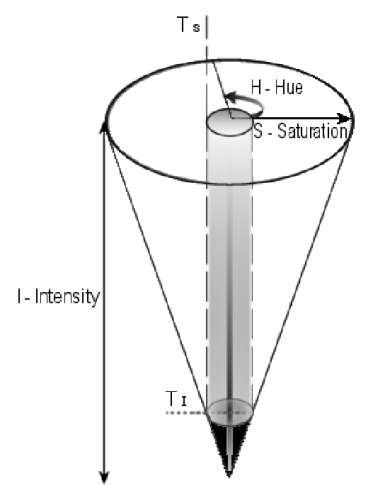


Therefore, in this paper a pixel $p$ will be characterised using the three-band colour representation given by the HSI colour space:

$$
\overrightarrow{f_{p}}=\left[H_{p}, S_{p}, I_{p}\right] .
$$

\subsection{Fuzzy resemblance between feature vectors}

In image segmentation, regions consist of a set of connected pixels whose features are resemblant, i.e., not different. Hence, we need to define resemblance between feature vectors, and a suitable tool for that purpose is the concept of fuzzy resemblance relation.

In general, we define a fuzzy resemblance relation between (real) feature vectors as a fuzzy subset $\mathcal{F R}$ of $\mathbb{R}^{n} \times \mathbb{R}^{n}$, with membership function

$$
\mathcal{F R}: \mathbb{R}^{n} \times \mathbb{R}^{n} \rightarrow[0,1]
$$

verifying the reflexive and symmetric properties, i.e., given two feature vectors $\overline{f_{1}}$ and $\overline{f_{2}}, \mathcal{F R}\left(\overline{f_{1}}, \overline{f_{1}}\right)=1$ and $\mathcal{F R}\left(\overline{f_{1}}, \overline{f_{2}}\right)=\mathcal{F R}\left(\overline{f_{2}}, \overline{f_{1}}\right)$.

The way resemblance between feature vectors is computed depends on the concrete features employed. Usually, resemblance between particular features or subvectors is calculated, depending on the specific feature domain, and then the resulting measures are aggregated. Finally, differences between subvectors of features may be aggregated using some appropriate aggregation function.

Resemblance between colours: In this paper the resemblance between pixel features will be measured by means of the resemblance between colour features, which will be calculated on the basis of distances in the HSI colour space.

Distance between colours in the HSI space

The distance between two colour stimuli $c_{1}=\left[H_{1}, S_{1}, I_{1}\right]$ and $c_{2}=\left[H_{2}, S_{2}, I_{2}\right]$ can be defined on the basis of the differences between its components:

$$
\begin{aligned}
& \Delta_{H}\left(c_{1}, c_{2}\right)= \begin{cases}\frac{\left|H_{1}-H_{2}\right|}{\pi} & \text { if }\left|H_{1}-H_{2}\right| \leq \pi \\
\frac{2 \pi-\left|H_{1}-H_{2}\right|}{\pi} & \text { otherwise }\end{cases} \\
& \Delta_{S}\left(c_{1}, c_{2}\right)=\left|S_{1}-S_{2}\right| \\
& \Delta_{I}\left(c_{1}, c_{2}\right)=\frac{\left|I_{1}-I_{2}\right|}{M A X I}
\end{aligned}
$$

with MAXI being a constant equalising to the maximum level of intensity, usually 255 (let us remark that $\Delta_{H}, \Delta_{S}, \Delta_{I} \in[0,1]$ ). Based on the previous distances, equation (5) will be used to measure the difference between colours (for the sake of simplicity, we have removed the parameters $\left(c_{1}, c_{2}\right)$ in the notation $\Delta_{H}, \Delta_{S}$, and $\left.\Delta_{I}\right)$. Notice that $\Delta C\left(c_{1}, c_{2}\right) \in[0,1]$. 


$$
\Delta C\left(c_{1}, c_{2}\right)= \begin{cases}\Delta_{I} & \text { if } p_{i} \text { or } p_{j} \text { are achromatic } \\ \frac{1}{\sqrt{3}}\left[\Delta_{I}^{2}+\Delta_{S}^{2}+\Delta_{H}^{2}\right]^{1 / 2} & \text { if } p_{i} \text { and } p_{j} \text { are chromatic } \\ \frac{1}{\sqrt{2}}\left[\Delta_{I}^{2}+\Delta_{S}^{2}\right]^{1 / 2} & \text { otherwise. }\end{cases}
$$

In equation (5) we introduce the notions of chromaticity/achromacitiy to manage two well-known problems of the HSI representation: the imprecision of the hue when the intensity or the saturation is small, and the non-representativity of saturation under low levels of intensity. An often practical solution to solve this problem is to perform a partition of the colour space based on the chromaticity degree of each point. In equation (5), we propose to split the HSI space into three regions: chromatic, semi-chromatic and achromatic (Figure 1) on the basis of thresholds $T_{I}$ and $T_{S}$ on the components $I$ and $S$ respectively. A colour $c_{i}=\left[H_{i}, S_{i}, I_{i},\right]$ will be achromatic if $I_{i} \leq T_{I}$ (black zone in Figure 1), semi-chromatic if $I_{i}>T_{I}$ and $S_{i} \leq T_{S}$ (grey zone in Figure 1), and chromatic if $I_{i}>T_{I}$ and $S_{i} \leq T_{S}$ (white zone in Figure 1). In this paper, the thresholds have been fixed empirically to $T_{I}=M A X I / 5$ and $T_{s}=1.5$.

\section{Resemblance between colours in the HSI space}

On the basis of the distance between colours given by the equation (5), we define the resemblance between the feature vectors $f_{p}$ and $f_{q}$ corresponding to pixels $p$ and $q$ of an image $I M$ as

$$
\mathcal{F R}\left(\overrightarrow{f_{p}}, \overrightarrow{f_{q}}\right)=1-\Delta C\left(\overrightarrow{f_{p}}, \overrightarrow{f_{q}}\right)
$$

where $\overrightarrow{f_{p}}=\left[H_{p}, S_{p}, I_{p}\right]$ and $\overrightarrow{f_{q}}=\left[H_{q}, S_{q}, I_{q}\right]$ are the colour features vector of $p$ and $q$ respectively.

\subsection{Fuzzy connectivity between pixels}

In fuzzy path-based image segmentation, the notion of fuzzy connectivity of two pixels indicates to which degree those pixels belong to a group of the topologically connected pixels with resemblant features. Because of this, to measure the fuzzy connectivity between two pixels we do not use directly the resemblance between their feature vectors, but we use information about the homogeneity of the paths joining them. Before entering into details, let us introduce some definitions.

Definition 2.1: A path between two pixels $p$ and $q$ is a sequence

$$
\pi_{p q}=\left(r_{1}, r_{2}, \ldots, r_{k}\right)
$$

where $k \geq 1$, such that $r_{1}=p$ and $r_{k}=q$ and $r_{i}$ is connected to $r_{i+1} \forall i \in\{1, \ldots, k-1\}$.

We note $\Pi_{p q}$ the set of possible paths linking the pixels $p$ and $q$ through pixels of the image $I M$. Also, we note $\mathcal{P}\left(\pi_{p q}\right)$ the set of pixels in the path $\pi_{p q}$ and $\pi_{p q}^{r s}$ the subpath of 
$\pi_{p q}$ that connects $r$ and $s$ with $r, s \in \mathcal{P}\left(\pi_{p q}\right)$, and where $r$ appears before $s$. Also, let $\pi_{p q}=\left\{p_{1}, \ldots, p_{n}\right\}$, then $\pi_{p q}^{-1}=\left\{p_{n}, \ldots, p_{1}\right\}$.

Definition 2.2: The fuzzy homogeneity of a path $\pi_{p q} \in \Pi_{p q}$, is defined as a function

$$
\text { homo }: \Pi_{p q} \rightarrow[0,1]
$$

calculated on the basis of the resemblances between consecutive points on the path.

To measure the resemblances between consecutive points, the relation $\mathcal{F R}$ will be used. In Section 3 we shall study desirable properties of homo and we shall propose some candidate functions.

Taking into account the homo function, we define the optimum path between $p$ and $q$, $\hat{\pi}_{p q}$, as the path that links both points with maximum homogeneity, in the following way.

Definition 2.3: The optimum path between $p$ and $q$ is:

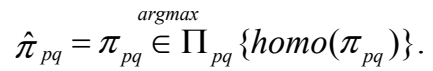

Based on this optimum path, we can get the measure of the connectivity between two pixels as follows.

Definition 2.4: The fuzzy connectivity between two pixels $p$ and $q$ is the homogeneity of the optimum path from $p$ to $q$ :

$$
\operatorname{conn}(p, q)=\operatorname{homo}\left(\hat{\pi}_{p q}\right)
$$

Let us remark that the homogeneity measure defined in equation (10) uses topographic information (paths linking the pixels) and resemblance between pixel features.

\subsection{Membership function for fuzzy regions}

In the above section we have introduced the use of paths to measure the fuzzy connectivity between any pair of pixels. Now this connectivity will be used to obtain fuzzy regions in an image $I M$ by means of the region growing technique showed in algorithm 1. Concretely, in this approach a region is defined as a fuzzy subset of connected pixels, so it is necessary to define a measure that indicates the degree in which each pixel in the image $I M$ belongs to each region. Under the assumption that a fuzzy region $\widetilde{R_{s}}$ has a representative seed point $r_{s}$ (as in the region growing techniques), we introduce the following membership function associated to each region.

Definition 2.5: The membership degree $\mu_{\widetilde{R}_{s}}(p)$ of a pixel $p$ in a fuzzy region $\widetilde{R_{s}}$ is defined as:

$$
\mu_{\overparen{R_{s}}}(p)=\operatorname{conn}\left(p, r_{s}\right)
$$

where $r_{s}$ is the seed point of $\widetilde{R_{s}}$. 
Using equation (11) we calculate the membership degree of every point $p \in I M$ to each region $\widetilde{R_{s}}$. It allows us to obtain a set of fuzzy regions $\widetilde{\Theta}=\left\{\widetilde{R}_{1}, \widetilde{R}_{2}, \ldots, \widetilde{R}_{m}\right\}$ from a set of seed points $\Theta=\left\{r_{1}, r_{2}, \ldots, r_{m}\right\}$.

Algorithm 1 summarises the steps to compute a fuzzy region $\widetilde{R}_{s}$. The algorithm starts at the seed point, $r_{s} \in \Theta$, which has maximum membership. In each iteration, the pixel nearest to the contour of the region is incorporated to it. The nearest pixel, $q$, is searched between all the neighbours of pixels in the contour of the region, and is the one with maximum resemblance regarding its neighbour in region's contour. In this way we obtain the optimum path for each pixel in the image to the given region's seed, with a computational cost of $O(n)$, where $n$ is the number of pixels in the image.

An algorithm for path-based fuzzy image segmentation only has to repeat the process in algorithm 1 for each seed. Therefore, the computational complexity of the fuzzy path-based segmentation algorithm is $O(m n), m$ being the number of seeds.

\section{Measuring the homogeneity of a path}

In previous section we have described a methodology for path-based fuzzy segmentation. In this section we discuss about possible ways to measure the homogeneity of a path.

\subsection{Path homogeneity intuition}

Given a path $\pi_{p q}=r_{1} \ldots r_{n}$. We want to come up with a function homo $\hat{\pi}_{p q}$ measuring its homogeneity. For the sake of simplicity, let us define a resemblance relation $\mathcal{P R}$ between neighbour pixels, induced by the relation $\mathcal{F R}$ between their corresponding features, in the following way:

$$
\mathcal{P R}(p, q)=\mathcal{F R}\left(\overline{f_{p}}, \overline{f_{q}}\right) .
$$

Hence, it seems natural to define homo $\left(\hat{\pi}_{p q}\right)$ as an aggregation of the resemblances between consecutive points in the path $\hat{\pi}_{p q}$, i.e., $\operatorname{homo}\left(\pi_{p q}\right)=\operatorname{Aggr}\left(\operatorname{ReSet}\left(\pi_{p q}\right)\right)$, where ReSet is the following bag (multiset) of values:

$$
\operatorname{ReSet}\left(\pi_{p q}\right)=\left\{\mathcal{P R}\left(r_{k}, r_{k+1}\right) \mid r_{k}, r_{k+1} \in \mathcal{P}\left(\pi_{p q}\right)\right\} .
$$

In order to choose the aggregation function Aggr, we study first the set of properties that the homo function should verify. We propose the following minimal set of properties for homo.

- Let $\pi_{p q}=p, q$ be a path consisting of two adjacent pixels. Then $\operatorname{homo}\left(\pi_{p q}\right)=\mathcal{P R}(p, q)$. As a consequence, if $\pi_{p p}=p, p$ then $\operatorname{homo}\left(\pi_{p p}\right)=1$.

- The homogeneity of a path should be less than or equal to the resemblance between consecutive pixels in the path, i.e., $h o m o\left(\pi_{p p}\right) \leq \min \operatorname{ReSet}\left(\pi_{p p}\right)$. The rationale behind this property is that a path is completely homogeneous if all the possible pairs of consecutive pixels are resemblant. Hence, the homogeneity of the path has an upper bound in the minimum value of resemblance between pairs of consecutive pixels. 
- Monotony: $\operatorname{homo}\left(\pi_{p q}^{r s}\right) \geq \operatorname{homo}\left(\pi_{p q}\right)$.

- $\quad$ Let $\pi_{p q}$ and $\pi_{p^{\prime} q^{\prime}}$ be two paths such that $\operatorname{ReSet}\left(\pi_{p q}\right)=\operatorname{Re} \operatorname{Set}\left(\pi_{p^{\prime} q^{\prime}}\right)$.

Then $\operatorname{homo}\left(\pi_{p q}\right)=\operatorname{homo}\left(\pi_{p^{\prime} q^{\prime}}\right)$. In particular, $\operatorname{homo}\left(\pi_{p q}\right)=\operatorname{homo}\left(\pi_{p q}^{-1}\right)$.

These properties suggest the use of a $t$-norm to aggregate the resemblances between consecutive pixels into the final homogeneity of the whole path, i.e., to define the aggregation function as

$$
\operatorname{Aggr}\left(\operatorname{ReSet}\left(\pi_{p q}\right)\right)=\Lambda \operatorname{ReSet}\left(\pi_{p q}\right)
$$

were $\wedge$ is a $t$-norm.

To show that this function satisfies all the properties we have required is of little importance.

\subsection{Some candidates}

The choice of the $t$-norm to be used in equation (14) depends on the application at hand. In order to discuss about this issue we have performed some experiments, where we have employed the following operators:

- Minimum

- $\quad$ Algebraic product $I_{A}(a, b)=a b$.

- $\quad$ Bounded difference $I_{B}(a, b)=\max (0, a+b-1)$.

- Dubois-Prade's parametric $t$-norm:

$$
I_{D P}(a, b)=\frac{a b}{\max (a, b, \alpha)}
$$

with $\alpha \in[0,1]$.

- Weber's parametric $t$-norm:

$$
I_{W}(a, b)=\max \left(0, \frac{a+b+\lambda a b-1}{1+\lambda}\right)
$$

with $\lambda>-1$.

- $\quad$ Frank's parametric $t$-norm:

$$
I_{F}(a, b)=\log _{s}\left(1+\frac{\left(s^{a}-1\right)\left(s^{b}-1\right)}{s-1}\right)
$$

with $s>0, s \neq 1$.

For the sake of comparison, we have chosen amongst the proposed, the last three operators (Dubois-Prade's, Weber's and Frank's parametric $t$-norms) because. 
- $\quad$ They are parametric $t$-norms, so we can study different aggregation alternatives by using different values in parameters of these well-known operators.

- We consider that in most of the cases, $t$-norms that yield values under those provided by the bounded difference are too strict because the resulting homogeneity values are too low in general, and hence significant membership values of fuzzy regions tends to be too low. Hence, we have chosen parametric operators that yield values between the minimum and the bounded difference $t$-norms.

In addition, we have considered the first three $t$-norms, though they are known to be particular cases of the other three, since they as well as their properties are very well known.

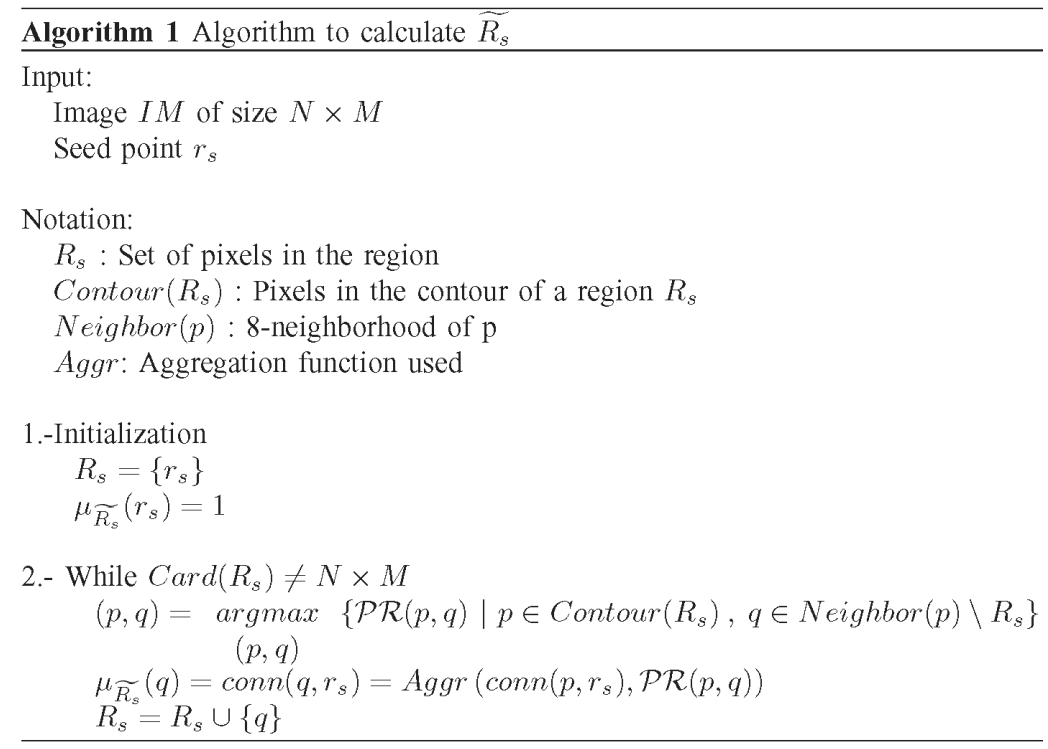

In the next section we show some experimental results in synthetic images as well as in real images, and we also discuss about using these $t$-norms, with several parameters, to calculate the homogeneity of a path. In addition we comment which of them should be used depending on the kind of region to segment.

\section{Experimental results}

Through experiments presented in this section we show the behaviour of our method in different situations.

\subsection{Synthetic images}

To test the behaviour of the aforementioned $t$-norms we have used synthetic images simulating different shades. Two of them, which serves to summarise our conclusions, are shown in Figures 2 and 3, where images of size $256 \times 256$ show a red toning down computed by means of Gaussian functions applied on the saturation component. 
Graphical representations in Figure 2-A1-2-A3 show the evolution of the homogeneity degree along the path indicated by a black line in Figure 2, computed with different aggregation functions. Specifically, for each $i \in\{1, \ldots, 255\}$ in the $X$ axis, the graphics show in the $Y$ axis the homogeneity degree of the subpath from $(0,0)$ to $(i, i)$.

Two main behaviours have been found among these $t$-norms. On one hand, we have functions with big slopes that reach the minimum homogeneity value in a point of the path close to the seed of the region. It means that all the pixels after this one have minimum membership degree, so the influence area of the region will be small. It produces regions where the fade is fast and strong at each bound, useful in regions with well defined contours or in those where it is necessary to find a frontier between regions whose bounds are fuzzy. This is the case of Weber $t$-norm when its parameter takes values under approximately -0.6 .

Figure 2 Results with a synthetic image generated by means of a Gaussian function simulating a toning down from a light incidence point

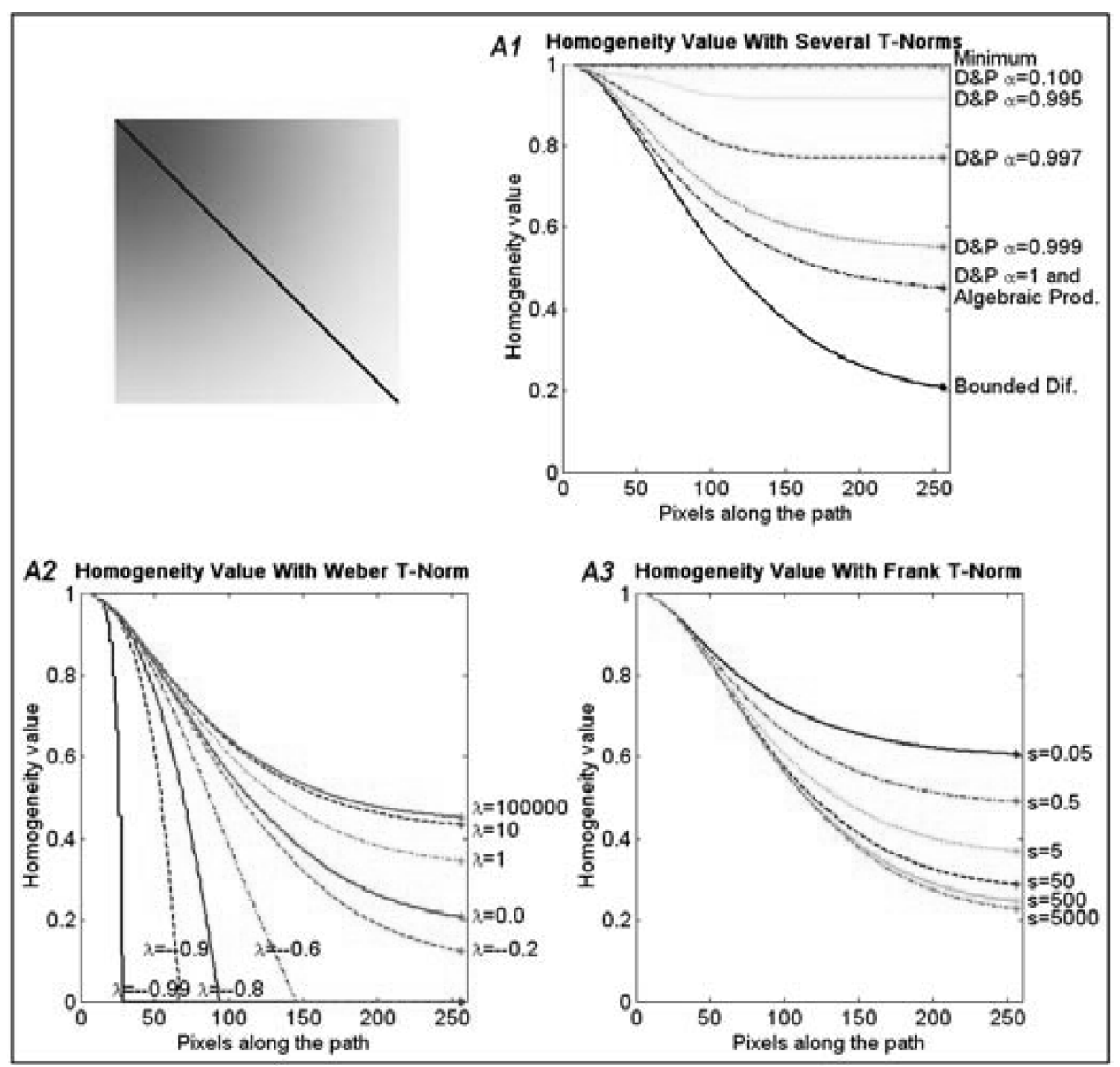


Figure 3 Results with a synthetic image generated by means of three Gaussian functions simulating toning down from three light incidence points

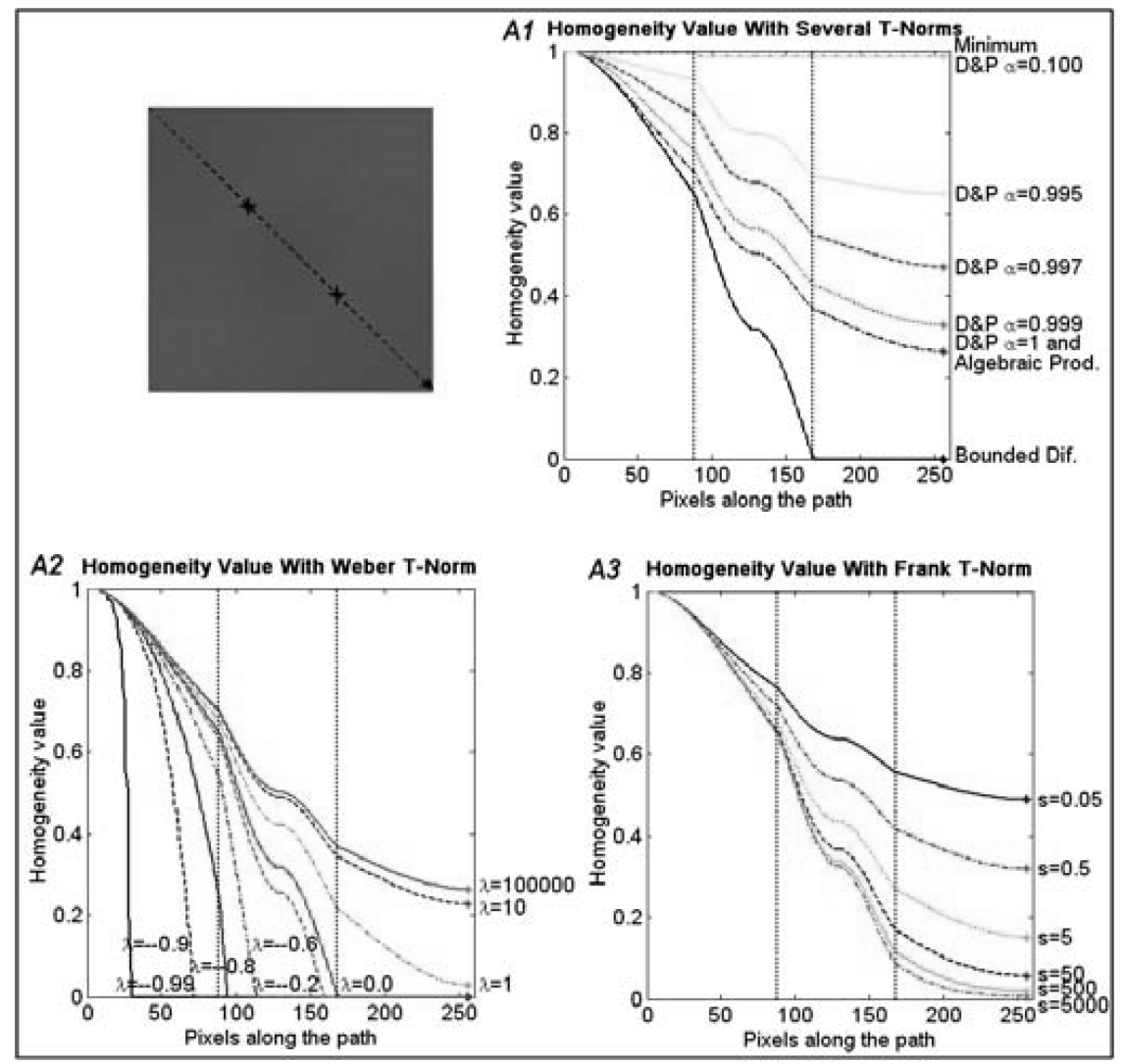

On the other hand, small slopes imply small and similar homogeneity values, so minimum homogeneity will only be reached in a pixel very far away from the origin of the region. The limit case is given by functions like minimum or Dubois-Prade $t$-norm with parameter under 0.997 , which have a point from which they are constant, so never reach minimum homogeneity. At the same time, homogeneity takes values in just a small part (the upper one) of the theoretical range. This kind of functions may be used, for example, in regions with a soft tone down where we want to have a slow decrease in membership value, letting the influence area of the seed extent through the whole region.

Finally, between both extreme behaviours there is a wide range of functions like, for example, Frank's $t$-norm, whose homogeneity curves for this example are showed in Figure 2-A3. In this case, when the parameter $s$ increase, the function assigns higher homogeneity values to pixels in the path and capture soft decreasing shades. Distance between the graphic representation of consecutive functions is smaller as $s$ grows.

Image in Figure 3 shows three soft shades in saturation component. Central points of the shades are in $(0,0),(128,128)$ and $(255,255)$. Again we study the homogeneity degree of the subpath from $(0,0)$ to $(i, i)$, marked as a black line in the image in Figure 3.

In this case we find that results are coherent regarding previous results, since $t$-norms keep the same behaviour aforementioned regarding their parameter, as Figure 3-A1-3-A3 shows. In addition, we observe that slope of all of the functions is 
higher in this image than for image in Figure 2, because of the higher strength of the three shades. In spite of this, we can also notice the stress the two main behaviours pointed before: soft and small slopes, so high homogeneity values, for Frank's $t$-norm (3-A2) and even more for Dubois-Prade $t$-norm (3-A1). On the other hand, Weber's $t$-norm (3-A3) has the biggest slopes, and so homogeneity decreases down to 0 around the middle of the path, for most of the values of its parameter.

Figure 3-A1-3-A3 show two vertical lines indicating the points where the curvature of the homogeneity functions change. These points, as expected, correspond to the pixels in the path, marked with crosses in the original image in Figure 3, where two shades meet. Finally, a third change in the curvature may be perceived: the origin of the central shade.

\subsection{Real images}

In these section we will show some examples of the usefulness of this variety of behaviours, applied to a real images shown in Figures 4-7. These images are representative of the kind of regions we may find in real images. All the types of regions we may find ranges between two extreme cases: on one hand, homogeneous regions with crisp contours, like in Figure 4; on the other hand fuzzy regions with fuzzy contours, like in Figure 7. Between these extreme cases we can find a variety of homogeneously coloured regions, whose contours become wider and fuzzier, as in Figures 5 and 6.

Figure 4 Results with a natural image, with well-defined contours

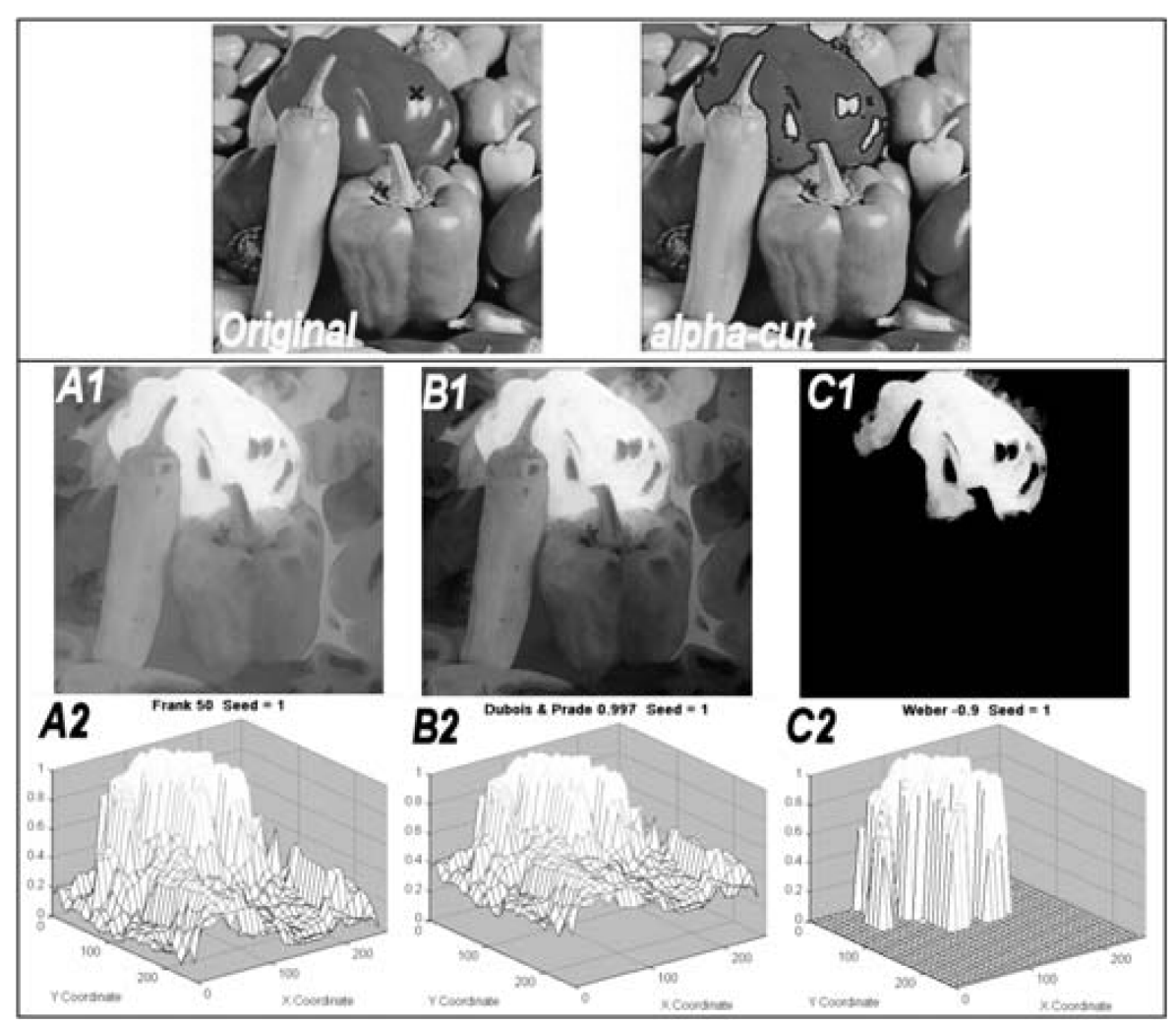


Figure 5 Results with a medical image with contours that are not completely well defined

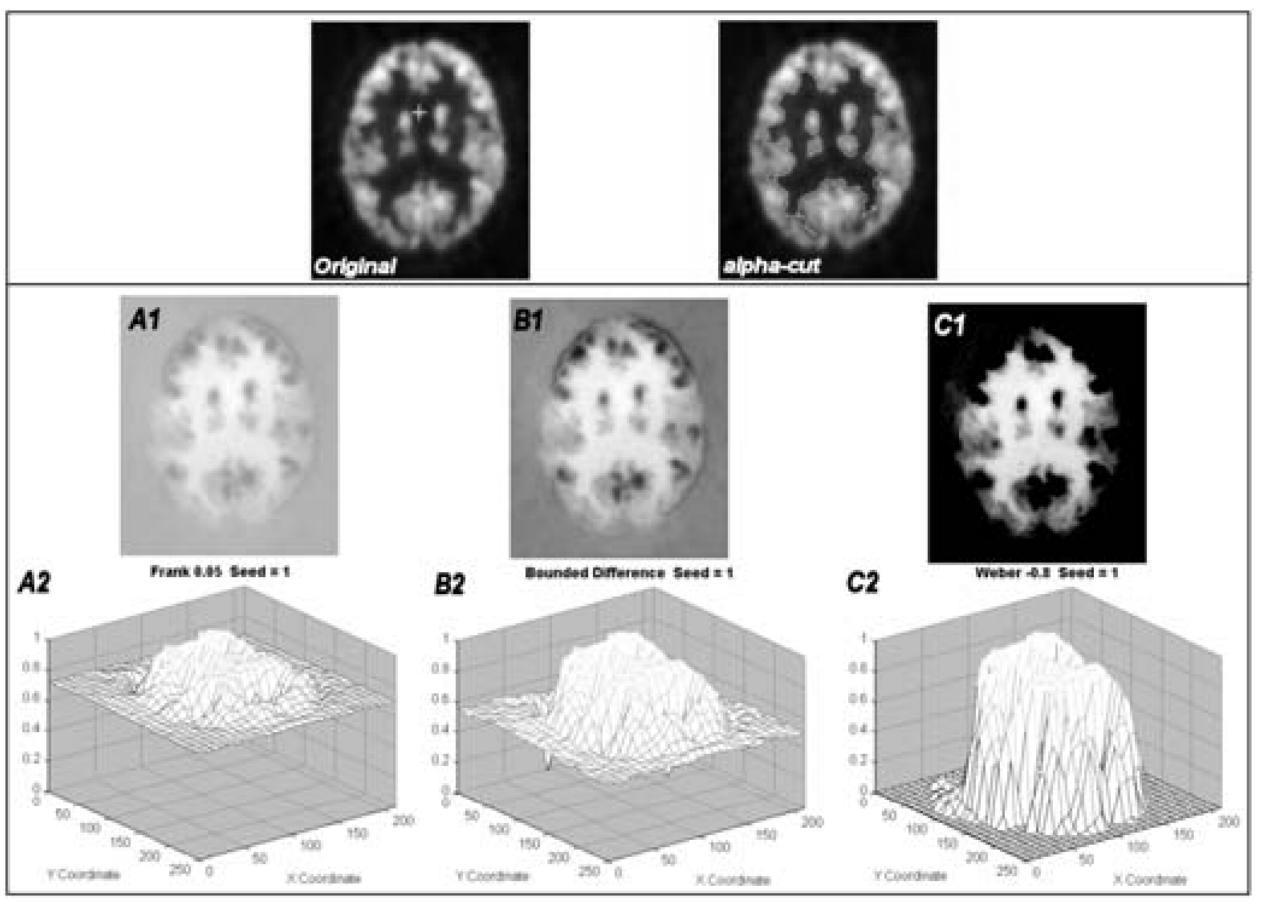

Figure 6 Results with a natural image, which is an homogeneous region with a fuzzy contour

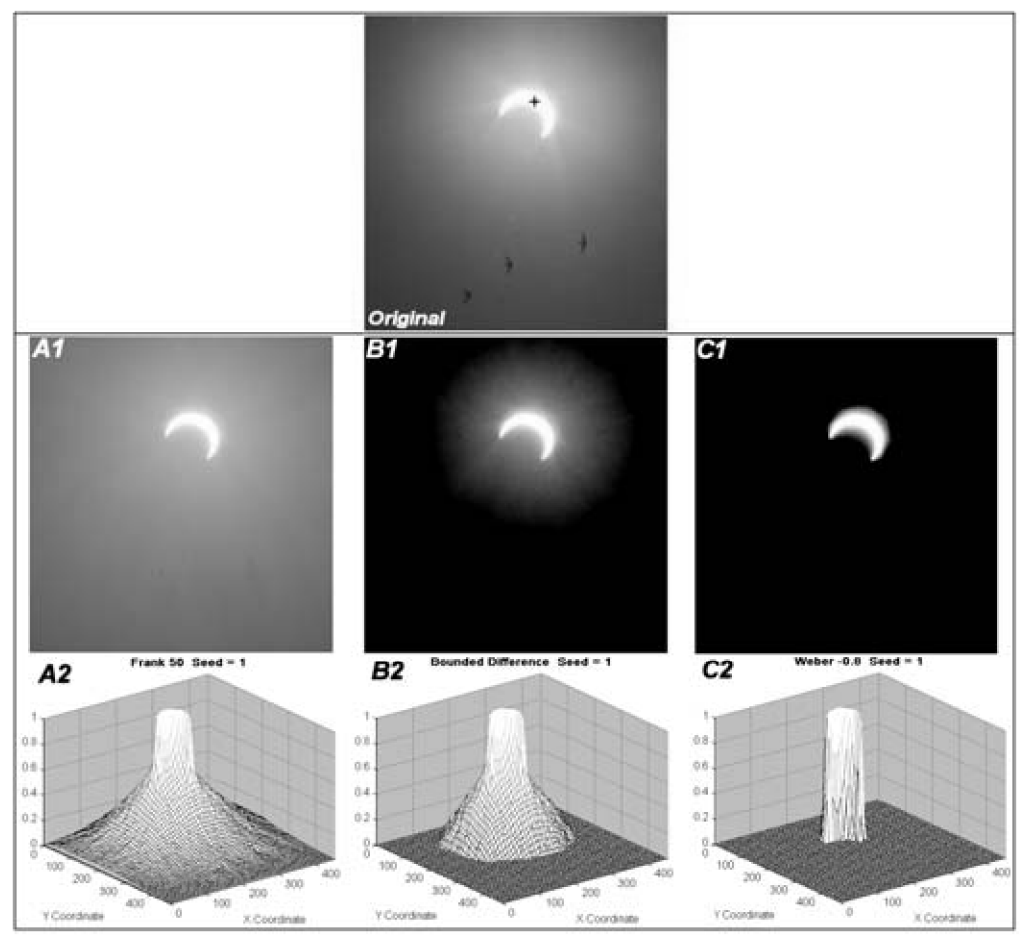


Figure 7 Results with an astrophysical image, corresponding to a fuzzy region with a fuzzy contour

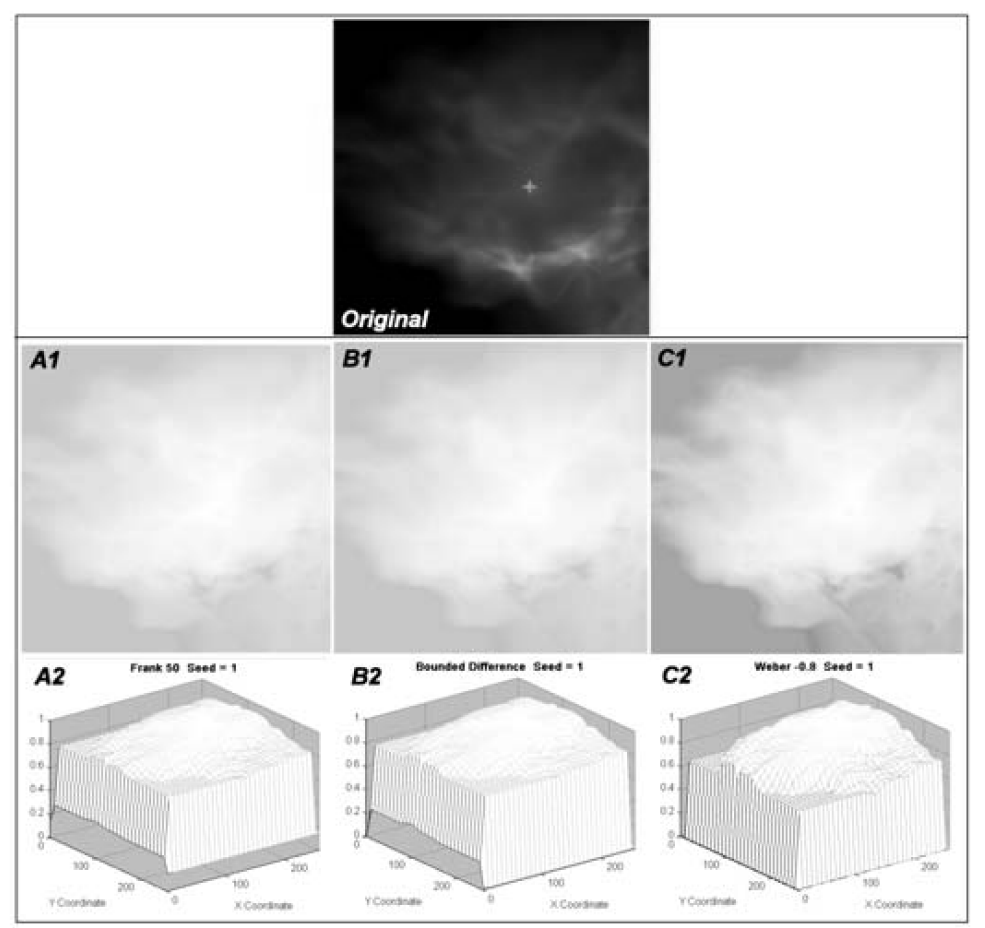

In all the figures in this section, the first row has the original image with the seed marked as a cross, while the second and third rows represent the membership value to a given seed, computed with a certain homogeneity function. In the second row, membership degrees are represented as grey levels; white colour means maximum membership value while black is the minimum. In the third one, a $3 \mathrm{D}$ representation of the region is shown, where the height of a point is its membership degree. Each of three columns, i.e., A, B and $\mathrm{C}$, corresponds to the results obtained using a given $t$-norm.

In the experiment showed in Figure 4, we have placed one seed in the red pepper, and we have computed the corresponding fuzzy region using different $t$-norms: Dubois-Prade $t$-norm with $\alpha=0.997$, Frank's with $s=50$, and Weber's with $\lambda=-0.9$ in columns A - C of Figure 4, respectively. Here, we have an homogeneous red region, with well defined contours and, as expected, we notice that the highest membership degrees correspond to the red round pepper, and also a significant decrease of membership values out of the boundary of the pepper. The magnitude of that decrease, which depends on the $t$-norm employed, can be better appreciated in the second row of images. We can also see how in Figure 4-C1, almost the whole area surrounding the pepper takes the membership value 0 , limiting the extent of the region to almost just the red round pepper. This is shown also in Figure 4-C2. In the other two cases, the support of the fuzzy region is almost the whole image, so they are less suitable to model the region.

In this case, since we have a well-defined region, it makes sense to look for an $\alpha$-cut bounding the area of the pepper. Since homogeneity function that best model the pepper region is Weber's $t$-norm, we have found on it the $\alpha$-cut in the first row for $\alpha \approx 0.7$. 
In this image, we have used some of the aforementioned membership functions to show that any of them may be used. In the next examples we have used Frank's $t$-norm with $s=0.05$ (A1,A2), Bounded difference $(\mathrm{B} 1, \mathrm{~B} 2)$ and Weber's $t$-norm with $\lambda=-0.8$ $(\mathrm{C} 1, \mathrm{C} 2)$ in all the cases, to be able to make comparisons.

Another real image example to illustrate the performance of different homogeneity functions is in Figure 5, that corresponds to an homogeneous regions with a not-well defined contours, wider and fuzzier than in the case of peppers image. Therefore, the function that best models is also Weber's $t$-norm, but with a parameter value that makes it softer than in the case of peppers. The $\alpha$-cut representing the region on this $t$-norm $(\alpha \approx 0.7$ ), is in the first row. Figure 5-C1, show how Weber function is, as expected, more restrictive in its consideration of homogeneity than the other $t$-norms. So with this function, dissimilarity between consecutive pixels produces a higher decrease in homogeneity values than with Bounded difference or Frank $t$-norm. As a consequence, the support of the fuzzy region computed with Weber is smaller than in the other cases: almost just the central dark orange region. It makes this function more suitable to detect the central area, since the other functions extents the fuzzy region over the orange bounds to the whole image. It is also interesting noticing what Figures 5-A2, B2 shows. In previous section we mentioned that the real range of homogeneity values is smaller for homogeneity functions with small slopes. This figure shows what it means at the time of segmentation: membership values vary in a small range, as the raised 3D graphic shows.

In the preceding examples, we have seen that homogeneity functions with big slopes in Figure 2, very sensitive to variability in the image, are suitable to model 'crisp' regions or regions with not too fuzzy contours. This is because in the moment the path goes out of an homogeneous area the homogeneity value abruptly decreases.

In the next two examples, it is shown that functions with small slopes are suitable for images with imprecise contours like in Figure 6 or even regions completely fuzzy like the one in Figure 7.

As can be seen in Figures 6-C1 and 7-C1, a high slope function will not be able to represent the whole fuzzy colour shade region. In this case a softer function would be more appropriated to adapt to the extent of the whole colour shade (in Figure 6) or the fuzzy region (in Figure 7). It is seen in images A1 in both figures: Figure 6-A1 shows how the support of the sun region extends through almost the whole tone down, while Figure 7-A1 shows how the fuzzy red nebula is better model by Frank $t$-norm, since softer in the transition from the region to the background.

\section{Conclusions}

As many intelligent applications demand, we have proposed a set of fuzzy measures that, combined with path-based fuzzy image segmentation techniques, lead us to obtain representations, by means of fuzzy sets, of the imprecision inherent to regions in images.

Path-based fuzzy image segmentation takes a colour image and yields a set of fuzzy regions, each region being a fuzzy subset of topologically connected pixels. As a way to determine membership degrees, we have proposed a fuzzy approach to calculate the homogeneity of a path. The homogeneity degree of a path is obtained as an aggregation of resemblances between colours of adjacent pixels in the path. Starting from a set of intuitive properties, we have chosen to employ $t$-norms as aggregation operators. 
We have studied the use of different $t$-norms that present a wide range of behaviours, giving us a powerful and flexible tool to adapt membership functions of fuzzy regions to any kind of colour image, depending on its purpose and characteristics.

In summary, two main different behaviours of $t$-norms have been found. On one hand, we have functions like Weber's functions with $\lambda \in(-1,-0.5]$, that are the most suitable ones when we are looking for homogeneous regions with relatively precise bounds (for example, unicellular organisms in biomedical images). On the other hand, functions like bounded difference, Dubois and Prade or Frank $t$-norm, are useful when bounds of the region are imprecise (for example, nebula images in astronomy, intercellular material in biomedical images, or toning down images like that seen in Figure 6). In general, from the order relation existing between $t$-norms, from minimum to the bounded difference, we obtain different slopes for the membership functions, allowing us to model all the types of regions between two extreme cases from regions with crisp contours to regions with fuzzy ones. These conclusions have been made after obtained studying several images from different environs.

As future work we shall study an automatic procedure to select the most suitable function depending on the characteristics of the regions in the image.

\section{References}

Borges, G. and Aldon, M. (2000) 'A split-and-merge segmentation algorithm for line extraction in $2 \mathrm{~d}$ range images', Proceedings of the 15th International Conference on Pattern Recognition, Vol. 1, pp.441-444.

Cavalho, B., Gau, C., Herman, G. and Kong, T. (1999) 'Algorithms for fuzzy segmentation', Proceedings of the International Conference on Advances in Pettern Recognition, Plymouth, UK, pp.154-163.

Chamorro-Martinez, J., Sanchez, D. and Prados-Suarez, B. (2003a) 'A fuzzy color image segmentation applied to robot vision', in Benitez, J.M., Cordon, O., Hoffman, F. and Roy, R. (Eds.): Advances in Soft Computing - Engineering, Design and Manufacturing, Springer, NY, USA, pp.129-138.

Chamorro-Martinez, J., Sanchez, D., Prados-Suarez, B., Galan-Perales, E. and Vila, M. (2003b) 'A hierarchical approach to fuzzy segmentation of colour images', IEEE International Conference on Fuzzy Systems, May, Vol. 2, St. Louis, Missouri, USA, pp.966-971.

Chen, T.Q. and Lu, Y. (2002) 'Color image segmentation - an innovative approach', Pattern Recognition, Vol. 35, pp.395-405.

Cheng, H.D. and Li, J. (2003) 'Fuzzy homogeneity and scale-space approach to color image segmentation', Pattern Recognition, Vol. 36, No. 7, pp.1545-1562.

Cheng, H.D., Jiang, X.H., Sun, Y. and Wang, J. (2001) 'Color image segmentation: advances and prospects', Pattern Recognition, Vol. 34, pp.2259-2281.

Chien, B-C. and Cheng, M-C. (2002) 'A color image segmentation approach based on fuzzy similarity measure', Proceedings of the 2002 IEEE International Conference on Fuzzy Systems, Hawaii, USA, May, Vol. 1, pp.449-454.

Gillet, A., Macaire, L., Botte-Lococq, C. and Postaire, J. (2001) 'Color image segmentation by fuzzy morphological transformation of the $3 \mathrm{~d}$ color histogram', Proceedings of the 10th IEEE International Conference on Fuzzy Systems, Vol. 2, p.824.

Hacbouf, F. and Mezhoud, N. (2003) 'An improved fuzzy rule-based segmentation system', Proceedings of the Seventh International Symposium on Signal Processing and its Applications, July 1-4, Vol. 1, pp.533-536.

Han, J. and Ma, K-K. (2002) 'Fuzzy color histogram and its use in color image retrieval', IEEE Transactions on Image Processing, Vol. 11, No. 8, pp.944-952. 
Maeda, J., Ishikawa, C., Novianto, S., Tadehara, N. and Suzuki, Y. (2000) 'Rough and accurate segmentation of natural color images using fuzzy region-growing algorithm', 15th International Conference on Pattern Recognition, Vol. 3, October, pp.638-641.

Makrogiannis, S., Vanhamel, I., Sahli, H. and Fotopoulos, S. (2001) 'Scale space segmentation of color images using watersheds and fuzzy region merging', Proceedings of the 2001 International Conference on Image Processing, Thessaloniki, Greece, Vol. 1, pp.734-737.

Moghaddamzadeh, A. and Bourbakis, N. (1997) 'A fuzzy region growing approach for segmentation of color images', Pattern Recognition, Vol. 30, No. 6, pp.867-881.

Ohno, T.M.S.Y.S. and Tsubouchi, K. (2001) 'A mobile robot campus walkway following with daylight-change-proof walkway color image segmentation', Proceedings of the IEEE International Conference on Intelligent Robots and Systems, Hawaii, USA, Vol. 1, pp.77-83.

Philipp-Foliguet, S., Viera, M.B. and Araujo, A.A. (2001) 'Segmentation into fuzzy regions using topographic distance', Proceedings of the XIV Brazilian Symposium on Computer Graphics and Image Processing, Florianóopolis, Brazil, pp.282-288.

Rosenfeld, A. (1979) 'Fuzzy digital topology', Information and Control, Vol. 40, No. 1, pp.76-87.

Russ, J. (1999) The Image Processing Handbook, 3rd ed., CRC Press and IEEE Press, Boca Raton, Florida.

Shiji, A. and Hamada, N. (1999) 'Color image segmentation method using watershed algorithm and contour information', Proceedings of International Conference on Image Processing, Kobe, Japan, Vol. 4, pp.305-309.

Udupa, J. and Samarasekera, S. (1996) 'Fuzzy connectedness and object definition: theory, algorithms and applications in image segmentation', Graphical Models and Image Processing, Vol. 58, No. 3, pp.246-261.

Yang, J-F., Hao, S-S. and Chung, P-C. (2002) 'Color image segmentation using fuzzy c-means and eigenspace projections', Signal Processing, Vol. 82, pp.461-472.

Zhong, D. and Yan, H. (2000) 'Color image segmentation using color space analysis and fuzzy clustering', Proceedings of the 2000 IEEE Signal Processing Society Workshop, Vol. 2, pp.624-633. 